\title{
Chiral symmetry breaking in gauged NJL model in curved spacetime
}

\author{
B. Geyer ${ }^{1}$ \\ Institute of Theoretical Physics and \\ Center for Theoretical Sciences \\ Leipzig University \\ Augustusplatz 10, D-04109 Leipzig, Germany \\ and \\ S. D. Odintsov ${ }^{2}$ \\ Dept. ECM, Fac. de Fisica, \\ Universidad de Barcelona, \\ Diagonal 647, 08028 Barcelona, Spain
}

\begin{abstract}
Using the renormalization group (RG) approach and the equivalency between the class of gauge-Higgs-Yukawa models and the gauged Nambu-Jona-Lasinio (NJL) model, we study the gauged NJL model in curved space-time. The behaviour of the scalar-gravitational coupling constant $\xi(t)$ in both theories is discussed. The RG improved effective potential of gauged NJL model in curved spacetime is found. The curvature at which chiral symmetry in the gauged NJL model is broken is obtained explicitly in a remarkably simple form. The powerful RG improved effective potential formalizm leads to the same results as ladder Schwinger-Dyson equations which have not been formulated yet in curved spacetime what opens new possibilities in the study of GUTs and NJL-like models in curved spacetime.
\end{abstract}

PACS numbers: 04.62.+v, 11.15.Pg, 11.30.Qc, 11.30.Rd, 04.60.-m.

1 E-mail: geyer@ntz.uni-leipzig.d400.de

2 E-mail: sergei@ecm.ub.es

On leave from Tomsk Pedagogical University, 634041 Tomsk, Russia 


\section{Introduction}

It is quite well known that dynamical symmetry breaking in the Standard Model (SM) may be realised via Nambu-Jona-Lasinio (NJL) mechanism [四], for example, with the help of quark condensate (see [2], [2a], [3] and references therein.) In such an approach - having in mind that in studying composite bound states the NJL model can be treated analytically - it is not by no means necessary to introduce elementary Higgs fields to discuss dynamical symmetry breaking.

A convenient way to study chiral symmetry breaking in the gauged NJL model is via Schwinger-Dyson (SD) equations, or equivalently, using the effective action for the composite fields [4], [5]. Very often it is necessary to study chiral symmetry breaking under some external conditions, like nonzero temperature, external electromagnetic or gravitational field. For example, the influence of the gravitational field on the chiral symmetry breaking may be important in the study of problems related to the early universe or to the physics of black holes.

In the past the study of the non-gauged NJL model in curved spacetime (in linear curvature approximation for which curvature effects actually may be relevant in GUT's and in SM) has shown quite rich phase structure and the possibility of curvature-induced transitions [6] between chiral symmetric and non-symmetric phases. Such a study has been done using the effective potential in the leading order of $1 / N$-expansion. Now, it is quite interesting to discuss similar questions for the more realistic gauged NJL model. This model recently has been studied in full detail using the ladder SD equations (see [7] where also renormalizability of gauged NJL model has been discussed). Unfortunately - due to the absence of the momentum representation in general curved spacetime - all attempts to generalize the SD equations to curved spacetime were not quite successful [8].

In the paper [3] (see also [9]) the NJL model has been discussed using the standard RG equations for coupling constants with appropriate compositeness conditions. This idea has been further elaborated in Ref. [10 for the gauged NJL model in flat spacetime. In particular, it has been shown that

some class of gauge Higgs-Yukawa models in leading order of a modified $1 / N_{c}$ expansion leads to a well-defined, non-trivial theory. This theory is

equivalent to the gauged NJL model when the ultraviolett cutoff goes to infinity (using corresponding compositeness conditions). 
In the present work we extend recent results of Harada, Kikukawa, Kugo and Nakano [10] on the study of the gauged NJL model to curved spacetime. In this way the RG-improved effective potential for the gauged NJL model in curved spacetime is found and its phase structure is discussed. The influence of the curvature to chiral symmetry breaking is described. Note that such an approach is proved to be equivalent to the ladder Schwinger- Dyson equations (at least in flat space) what maybe useful for generalization of SchwingerDyson equations to curved spacetime.

The paper is organized as follows: In Section 2 a brief review of the model and the approximation used in Ref. [10 is given. In Section 3 the gauge Higgs-Yukawa model is discussed in curved spacetime. In particular, the RG equation for scalar-gravitational coupling constant $\xi(t)$ and the oneloop effective potential are found. In Section 4, using the running couplings of the two previous sections and the approach of [3], [10] the RG-improved effective potential in gauged NJL model in curved spacetime is found (in linear curvature approximation). In the case of fixed gauge coupling the conditions for chiral symmetry breaking with account of the curvature are derived explicitly. Some remarks concerning the extension of our results are given in the Conclusion.

\section{Review of the class of gauge Higgs-Yukawa models}

In this section we review the class of gauge Higgs-Yukawa models in flat spacetime we are going to extend and the approximations we are using in such an analysis. Thereby we closely follow Ref. [10] where, using the RG approach, this model has been considered in detail.

The Lagrangian of the model in flat spacetime is given by (we use the notations of [10])

$$
\begin{aligned}
L_{m}= & -\frac{1}{4} G_{\mu \nu}^{a} G^{a \mu \nu}+\frac{1}{2}\left(\partial_{\mu} \sigma\right)^{2}-\frac{1}{2} m^{2} \sigma^{2}-\frac{\lambda}{4} \sigma^{4} \\
& +\sum_{i=1}^{N_{f}} \bar{\psi}_{i} i \hat{\mathcal{D}} \psi_{i}-\sum_{i=1}^{n_{f}} y \sigma \bar{\psi}_{i} \psi_{i} .
\end{aligned}
$$

Here the gauge group $S U\left(N_{c}\right)$ is chosen, with $N_{f}$ fermions $\psi_{i}\left(i=1,2, \ldots, N_{f}\right)$ belonging to the representation $R$ of $S U\left(N_{c}\right), \sigma$ is the scalar field. 
Let us now describe the $1 / N$ approximation for the perturbative study of this theory at high energies through the RG equations:

a) The gauge coupling constant is assumed to be small:

$$
\frac{g^{2} N_{c}}{4 \pi}<<1
$$

and the RG equations are considered only in the first nontrivial order on $g^{2}$. b) The number of fermions should be large enough:

$$
N_{f} \sim N_{c} \text {. }
$$

On the same time it is supposed that only $n_{f}$ fermions $\left(n_{f} \ll N_{f}\right)$ have large Yukawa couplings, whereas the remaining fermions have vanishing Yukawa couplings.

c) The approach is assumed to be perturbative also in $1 / N_{c}$, so only the leading order of the $1 / N_{c}$ expansion survives; this means that scalar loop contributions should be negligible

$$
\left|\frac{\lambda}{y^{2}}\right| \leq N_{c}
$$

It is interesting to note that for the minimal SM we have $N_{c}=N_{f} / 2=3$ and $n_{f}=1$.

Within the above approximation the RG equations for the coupling constants in (1) are [1], [12]:

$$
\begin{aligned}
& \frac{d g(t)}{d t}=-\frac{b}{(4 \pi)^{2}} g^{3}(t) \\
& \frac{d y(t)}{d t}=\frac{y(t)}{(4 \pi)^{2}}\left[a y^{2}(t)-c g^{2}(t)\right], \\
& \frac{d \lambda(t)}{d t}=\frac{u y^{2}(t)}{(4 \pi)^{2}}\left[\lambda(t)-y^{2}(t)\right] .
\end{aligned}
$$

where $b=\left(11 N_{c}-4 T(R) N_{f}\right) / 3, c=6 C_{2}(R), a=u / 4=2 n_{f} N_{c}$. For the fundamental representation we have $T(R)=1 / 2, C_{2}(R)=\left(N_{c}^{2}-1\right) /\left(2 N_{c}\right) \cdot t=$ $\ln \left(\mu / \mu_{0}\right)$ and $\mu_{0}$ is the reference scale to discuss low energy physics.

The RG equation for $g^{2}$ (respectively $\alpha=g^{2} / 4 \pi$ ) is solved by 


$$
\eta(t) \equiv \frac{g^{2}(t)}{g_{0}^{2}} \equiv \frac{\alpha(t)}{\alpha_{0}}=\left(1+\frac{b \alpha_{0}}{2 \pi} t\right)^{-1} .
$$

To solve the RG equations for the Yukawa and the scalar couplings it is convenient to introduce the following $\mathrm{RG}$ invariants:

$$
\begin{aligned}
& h(t) \equiv-\eta^{-1+c / b}(t)\left[1-\frac{c-b}{a} \frac{g^{2}(t)}{y^{2}(t)}\right], \\
& k(t) \equiv-\eta^{-1+2 c / b}(t)\left[1-\frac{2 c-b}{2 a} \frac{\lambda(t)}{y^{2}(t)} \frac{g^{2}(t)}{y^{2}(t)}\right] .
\end{aligned}
$$

With their help we obtain

$$
\begin{aligned}
& y^{2}(t)=\frac{c-b}{a} g^{2}(t)\left[1+h_{0} \eta^{1-c / b}(t)\right]^{-1}, \\
& \lambda(t)=\frac{2 a}{2 c-b} \frac{y^{4}(t)}{g^{2}(t)}\left[1+k_{0} \eta^{1-2 c / b}(t)\right] .
\end{aligned}
$$

Of course, these solutions are explicitly known only when the values of the $\mathrm{RG}$ invariants $h$ and $k$ are given.

As it has been shown in detail in Ref. [10] for the solutions (6) - (8) to be consistent with the above approximation $(2)-(4)$, and in order for the theory to be non-trivial one, we should have (see also related discussion in []])

$$
c>b \text { and } 0 \leq h_{0}<\infty \text {; }
$$

that makes the Yukawa coupling to be asymptotically free and the theory to be non-trivial (see also Ref. [13]). Further analysis shows that the scalar coupling constant is nontrivial in the above approximation only if

$$
k_{0}=0 \text {. }
$$

Below we will discuss the solutions (8) with conditions (9) and (10) only. The case $k_{0}=h_{0}=0, c>b$ actually corresponds to the fixed point of Ref. [14]. Obviously, in this case a reduction of couplings takes place [15], [16]. However, one can get also another fixed point [17] in gauged NJL model (see the discussion in [3]). 


\section{Gauge Higgs-Yukawa model in curved spacetime}

Let us extend now the model of the previous section to curved spacetime. It is by now well-known (see Ref. [18] for an introduction) that in order to be multiplicatively renormalizable in curved spacetime, the Lagrangian of the theory should be:

$$
L=L_{m}+L_{e x t}-\frac{1}{2} \xi R \sigma^{2},
$$

where $L_{m}$ is given by (1) with a change of flat (partial) derivatives to the corresponding covariant derivatives: $\partial_{\mu} \rightarrow \nabla_{\mu}$, $\xi$ is the non-minimal scalargravitational coupling constant, and

$$
L_{\text {ext }}=a_{1} R^{2}+a_{2} C_{\mu \nu \alpha \beta}^{2}+a_{3} G+a_{4} \square R+\Lambda-\frac{1}{\kappa} R .
$$

The Lagrangian $L_{\text {ext }}$ of the external gravitational field is necessarily introduced in order to have the theory to be multiplicatively renormalizable one; $a_{1}, \ldots a_{4}, \kappa$ are gravitational coupling constants.

Since the RG equations for those coupling constants which are present in flat space do not change in curved spacetime [18], all the discussions of Section 2 (as well as the approximations introduced there) are also valid here. In addition, the effective coupling constants corresponding to $\xi, a_{1}, \ldots, a_{4}, G$ appear. However, for our purposes, we only need the $R G$ equation for the coupling constant $\xi$.

The technique to calculate the one-loop $\beta$-function of $\xi$ is quite wellknown [18]; therefore, we will give only the final answer (within the above described approximations):

$$
\frac{d \xi(t)}{d t}=\frac{1}{(4 \pi)^{2}} 2 a y^{2}(t)\left(\xi(t)-\frac{1}{6}\right)
$$

Taking into account that

$$
\frac{d}{d t} f(t) \equiv \frac{d}{d t}\left\{\eta^{c / b}(t) \frac{\left(\xi(t)-\frac{1}{6}\right)}{y^{2}(t)}\right\}=0
$$

we may use this RG invariant $f(t)$ to find the solution of (13):

$$
\xi(t)=\frac{1}{6}+y^{2}(t) \eta^{-c / b}(t) f_{0}
$$


where $f_{0} \neq 0$ is the value of the RG invariant. Note that in the UV-limit $(t \rightarrow \infty)$ we have:

$$
\begin{aligned}
& y^{2}(t) \sim \frac{c-b}{a} \frac{g_{0}^{2}}{h_{0}} \eta^{c / b}(t) \rightarrow+0 \quad\left(h_{0} \geq 0\right) \\
& \xi(t) \simeq \frac{1}{6}+\frac{c-b}{a} g_{0}^{2} \frac{f_{0}}{h_{0}} .
\end{aligned}
$$

For $f_{0} \approx 0$ the scalar-gravitational coupling constant $\xi(t) \rightarrow 1 / 6$, i.e. asymptotic conformal invariance is realized [19. Hence, the RG invariant $f_{0}$ characterizes the deviation from conformal invariance $(\xi=1 / 6)$. Different types of UV-behaviour of $\xi(t)$ for different GUT's have been listed in [18]; the most typical ones are:

$$
\begin{aligned}
& \text { a) } \xi(t) \rightarrow \frac{1}{6} \text { [18], 19, } \\
& \text { b) }|\xi(t)| \rightarrow \infty \text { 18, 19, } \\
& \text { c) } \xi(t) \rightarrow \xi \text { 18. }
\end{aligned}
$$

As we can see, unlike the analysis in the case of flat spacetime, there will not appear any restrictions to the sign and the value of $f_{0}$ from the study of the UV-asymptotics of $\xi(t)$.

Let us discuss now the one-loop effective potential for the theory (1) in the approach where we keep only terms with the accuracy up to linear curvature [20], i.e. $\sigma^{2} \gg|R|$. Using the technique of Ref. [20] and working in leading order of the approximation (2) - (4), after some calculations one gets:

$$
\begin{aligned}
V= & \frac{1}{2} m^{2} \sigma^{2}+\frac{\lambda}{4} \sigma^{4}+\frac{1}{2} \xi R \sigma^{2}-\frac{a \mu_{F}^{4}}{2(4 \pi)^{2}}\left[\ln \frac{\mu_{F}^{2}}{\mu^{2}}-\frac{3}{2}\right] \\
& -\frac{a R \mu_{F}^{2}}{12(4 \pi)^{2}}\left[\ln \frac{\mu_{F}^{2}}{\mu^{2}}-1\right],
\end{aligned}
$$

where $\mu_{F} \equiv y \sigma$.

Using such a form for the potential $V$ one may investigate the curvatureinduced phase transitions which have been discussed in detail in Refs. [18], 20] for different GUT's. 


\section{Gauged Nambu-Jona Lasinio model in curved space- time}

We now discuss the gauged NJL model in curved spacetime. This model is quite popular due to different reasons, for example, the dynamical symmetry breaking in NJL models may also be relevant for the electroweak interaction with the top quark condensate as an order parameter (see [2], [3]), NJL-like theories maybe used for equivalent descriptions of the Standard Model [5], [21]. Moreover, the renormalizability of gauged NJL model may be proven in some sense [10] (for earlier discussions on renormalizability of the NJL model see [22]).

Under the assumption that not elementary ones but composite bound states are mainly relevant in quantum cosmology (for a recent discussion see [23]), there was an attempt to study non-gauged NJL model in curved spacetime [9], [6] and, in particular, to consider the chiral symmetry breaking under the influence of the external gravitational field [6]. Such an investigation has been done via explicit calculation of the effective potential for the composite field $\langle\bar{\psi} \psi>$. Our purpose here will be to discuss the same question on RG language for the gauged NJL model using its equivalency with the gauged Higgs-Yukawa model (1) (the explicit loop calculations in such a model are extremely hard to do).

We start from the gauged NJL model with four-fermion coupling constant $\mathrm{G}$ in curved spacetime

$$
L=-\frac{1}{4} G_{\mu \nu}^{2}+\sum_{i=1}^{N_{f}} \bar{\psi} i \hat{\mathcal{D}} \psi_{i}+G \sum_{i=1}^{n_{f}}\left(\bar{\psi}_{i} \psi_{i}\right)^{2} .
$$

As usually one introduces an auxiliary field $\sigma$ to replace the NJL model by the equivalent Higgs-Yukawa model. As is shown in Ref. [3], it is possible within the RG approach to use a set of boundary conditions for the effective couplings of the gauge Higgs-Yukawa model at $t_{\Lambda}=\ln \left(\Lambda / \mu_{0}\right)$ (where $\Lambda$ is the UV-cutoff of the gauged NJL model) in order to identify the gauged NJL model with the gauge Higgs-Yukawa model. Taking into account Eqs. (8) the explicit expressions for these compositeness conditions (which are specified in [3]) are given as (see also Eqs. (4.7) and (4.8) of [10]): 


$$
\begin{aligned}
& y^{2}(t)=\frac{c-b}{a} g^{2}(t)\left[1-\left(\frac{\alpha(t)}{\alpha\left(t_{\Lambda}\right)}\right)^{1-c / b}\right]^{-1} \equiv y_{\Lambda}^{2}(t), \\
& \frac{\lambda(t)}{y^{4}(t)}=\frac{2 a}{2 c-b} \frac{1}{g^{2}(t)}\left[1-\left(\frac{\alpha(t)}{\alpha\left(t_{\Lambda}\right)}\right)^{1-2 c / b}\right] \equiv \frac{\lambda_{\Lambda}(t)}{y_{\Lambda}^{4}(t)},
\end{aligned}
$$

where $t<t_{\Lambda}$. Hence, the system (1) with cuttoff $\Lambda$ is equivalent to the system (19) with the same cutoff when the running coupling constants are given by (20). In this sense the gauged NJL model may be called a renormalizable one. In addition, as it has been shown in [10], one should have also a compositeness condition for the mass, whose most convenient form will be (for $b \rightarrow+0$; [10]):

$$
m^{2}(t)=\frac{2 a}{(4 \pi)^{2}}\left(\frac{\Lambda^{2}}{\mu^{2}}\right)^{w} y_{\Lambda}^{2}(t) \mu^{2}\left[\frac{1}{g_{4}(\Lambda)}-\frac{1}{w}\right],
$$

where $G \equiv\left((4 \pi)^{2} / a\right) g_{4}(\Lambda) / \Lambda^{2}, g_{4}(\Lambda)$ is a dimensionless constant, and $w \equiv$ $1-\alpha /\left(2 \alpha_{C}\right), \alpha \equiv \alpha_{0}$ and where $y_{\Lambda}^{2}(t)$ is given by the first of Eqs. (20) at $b \rightarrow+0$.

The above compositeness conditions define the gauged NJL model as the gauge Higgs-Yukawa model in flat spacetime. Now, since we are working in curved spacetime (in linear curvature approximation), one has to add the compositeness condition for $\xi\left(t_{\Lambda}\right)$. Making the calculations in the same way as in [9] one obtains

$$
\xi\left(t_{\Lambda}\right)=\frac{1}{6}
$$

Obviously, the compositeness condition for $\xi(t)$ is again the same one as in the non-gauged NJL model [9]. Hence, the conformal invariance due to the renormalization group [19] takes place again. Analysing the RG equation (13) for $\xi(t)$ we will find that in all cases (i.e. for the general situation, where $y(t)$ is given by $(20)$, for the case with $b \rightarrow 0$ and with the corresponding $y(t)$, and also for the case $\alpha_{0} \rightarrow 0$, i.e. the non-gauged NJL model) we have

$$
\xi(t)=\frac{1}{6}
$$

In particular, for $b \rightarrow 0$ in the limit $\Lambda \rightarrow 0$ the fixed point of Ref. [14] in curved spacetime is given by 


$$
y_{*}^{2}=\frac{(4 \pi)^{2} \alpha}{2 a \alpha_{C}}, \quad \frac{\lambda_{*}}{y_{*}^{4}}=\frac{2 a}{(4 \pi)^{2}} \frac{\alpha_{C}}{\alpha}, \quad \xi_{*}=\frac{1}{6} .
$$

Now, one can study the possibility of chiral symmetry breaking in the model under discussion using the effective potential language, or more precisely, the RG-improved effective potential (see [24, 25] and references therein). Because the technique to study the RG-improved effective potential is widely known for the flat space [24], [25] as well as for curved spacetime [20] we will not give any details of its derivation.

Using the fact that the effective potential satisfies the $R G$ equation, one can explicitly solve this equation by the method of characteristics, and we find (for more details, see [24, 25] and [20]):

$$
V\left(g, y, \lambda, m^{2}, \xi, \sigma, \mu\right)=V\left(g(t), y(t), \lambda(t), m^{2}(t), \xi(t), \sigma(t), \mu e^{t}\right),
$$

where the effective coupling constants $g(t), \ldots, \sigma(t)$ are defined by the RG equations (5), (13) (at the scale $\mu e^{t}$ ) and corresponding RG equations for $m^{2}(t), \sigma(t)$ written in [10] for the case of the above gauge-Higgs-Yukawa model ( $t$ is left unspecified for the moment). As boundary condition it is convenient to use the one-loop effective potential (18).

In the case of the gauged NJL model one has to substitute into the effective potential (18) the effective couplings fulfilling compositeness conditions in accordance with Eqs. (25). In this way, one obtains the RG improved effective potential in the gauged NJL model. In flat spacetime such calculation has been already done in Ref. [10] for the case of fixed gauge coupling $(b \rightarrow+0)$, so we may use for the running coupling constants (except scalar-gravitational coupling constant) the results which are known from flat spacetime calculations.

Taking into account that the condition

$$
\mu e^{t}=\mu_{F}(t)
$$

serves actually for finding $t$, we get for the case of fixed gauge coupling

$$
e^{t}=\left(\frac{\mu_{F}(\mu)}{\mu}\right)^{1 /(2-w)}
$$


where $\mu_{F}(\mu)=\mu_{F}(t=0)$. Using (20), (23) and (27) in the case $b \rightarrow 0$ one gets (for the case of flat space see expression (6.13) of [10]):

$$
\begin{aligned}
\frac{(4 \pi)^{2}}{2 a} \frac{V}{\mu^{4}}= & \frac{1}{2} y_{\Lambda}^{2}(\mu)\left(\frac{\Lambda^{2}}{\mu^{2}}\right)^{w}\left(\frac{1}{g_{4}(\Lambda)}-\frac{1}{g_{4}^{*}}\right) \frac{\sigma^{2}(\mu)}{\mu^{2}} \\
& +\frac{\alpha_{C}}{4 \alpha}\left[\left(\frac{y_{\Lambda}(\mu) \sigma(\mu)}{\mu}\right)^{\frac{4}{2-w}}-\left(\frac{\mu^{2}}{\Lambda^{2}}\right)^{\frac{\alpha}{\alpha_{C}}}\left(\frac{y_{\Lambda}(\mu) \sigma(\mu)}{\mu}\right)^{4}\right] \\
& +\frac{3}{8}\left(\frac{y_{\Lambda}(\mu) \sigma(\mu)}{\mu}\right)^{\frac{4}{2-w}} \\
& +\frac{R}{24 \mu^{2}}\left(\frac{y_{\Lambda}(\mu) \sigma(\mu)}{\mu}\right)^{\frac{2}{2-w}}\left(1+\frac{2 \alpha_{C}}{\alpha}\right) \\
& -\frac{R \alpha_{C}}{12 \alpha \mu^{2}}\left(\frac{\mu}{\Lambda}\right)^{\frac{\alpha}{\alpha_{C}}} \frac{y_{\Lambda}^{2}(\mu) \sigma^{2}(\mu)}{\mu^{2}}
\end{aligned}
$$

where $g_{4}^{*} \equiv w$. Thus, we have got the RG-improved effective potential for the gauged NJL model (with finite cutoff) in curved spacetime.

Taking the limit $\Lambda \rightarrow \infty$ we will get the renormalized effective potential of the gauged NJL model in curved spacetime:

$$
\begin{aligned}
\frac{(4 \pi)^{2}}{2 a} \frac{V}{\mu^{4}}= & \frac{1}{2}\left(\frac{1}{g_{4 R}(\mu)}-\frac{1}{g_{4 R}^{*}}\right) \frac{y_{*}^{2} \sigma^{2}(\mu)}{\mu^{2}}+ \\
& +\frac{\alpha_{C}}{4 \alpha}\left(1+\frac{3 \alpha}{2 \alpha_{C}}\right)\left(\frac{y_{*} \sigma(\mu)}{\mu}\right)^{\frac{4}{2-w}} \\
& +\frac{R}{24 \mu^{2}}\left(1+\frac{2 \alpha_{C}}{\alpha}\right)\left(\frac{y_{*} \sigma(\mu)}{\mu}\right)^{\frac{2}{2-w}}
\end{aligned}
$$

where $y_{*}$ is given by (24) and the four-fermion coupling renormalization has been done; in particular $g_{4 R}^{*}$ is a finite constant (for discussion of that renormalization see [10], [7]).

Note that in flat space $(R=0)$ the potential $(29)$ in the same approach has been obtained in Ref. [7] (for non-renormalized potential see also [26]) using the ladder SD equation. Hence, the RG-improved effective potential may serve as a very useful tool to study the non-perturbative effects on 
equal footing with the SD equation. It is really surprising that RG improved effective potential gives the same results as ladder SD equation.

Using the finite effective potential (29) we are able to discuss the chiral symmetry breaking in the gauged NJL model under consideration. In flat spacetime, the possibility of chiral symmetry breaking is defined by the sign of the first term in (29). That gives the value of the ciritical four-fermion coupling constant $g_{4}^{*}(\Lambda)=w$. In curved spacetime the situation is more complicated as one has additional terms in the effective potential (29), even in the linear curvature approximation.

Taking into account that

$$
\begin{aligned}
& w=1-\frac{3 C_{2}(R)}{2(2 \pi)^{2}} g^{2} \simeq 1-\frac{3}{4 \pi} \frac{N_{c} g^{2}}{4 \pi} \simeq 1, \\
& \frac{\alpha_{C}}{\alpha} \simeq \frac{2 \pi}{3} \frac{4 \pi}{N_{c} g^{2}}>>1
\end{aligned}
$$

and introducing $x=y_{*} \sigma(\mu) / \mu$, one can rewrite the quadratic part of the potential (29) as follows:

$$
\frac{(4 \pi)^{2}}{2 a} \frac{V^{(2)}}{\mu^{4}} \simeq\left\{\frac{1}{2}\left(\frac{1}{g_{4 R}(\mu)}-\frac{1}{g_{4 R}^{*}}\right)+\frac{R}{12 \mu^{2}} \frac{\alpha_{C}}{\alpha}\right\} x^{2} .
$$

Relation (31) determines the way to estimate the chiral symmetry breaking for the gauged NJL model in curved spacetime.

In particular, in flat spacetime and for (cutoff) dependent effective potential we observe that the chiral symmetry is broken for

$$
\frac{1}{g_{4}(\Lambda)}-\frac{1}{g_{4}^{*}}<0 \text { or } \frac{1}{g_{4 R}(\mu)}-\frac{1}{g_{4 R}^{*}}<0
$$

Hence, the critical value of the four-fermion coupling constant which divides the chiral symmetric and non-symmetric phase is $g_{4}^{*}=w$.

In curved spacetime, chiral symmetry is always broken if

$$
\left(\frac{1}{g_{4 R}(\mu)}-\frac{1}{g_{4 R}^{*}}\right)+\frac{\alpha_{C}}{6 \alpha} \frac{R}{\mu^{2}}<0 \text {. }
$$


When this condition (33) is valid one can easily find the curvature-induced dynamical fermion mass.

For the cutoff-dependent effective potential the corresponding condition looks as:

$$
\left(\frac{\Lambda^{2}}{\mu^{2}}\right)^{w}\left(\frac{1}{g_{4}(\Lambda)}-\frac{1}{g_{4}^{*}}\right)+\frac{R}{6 \mu^{2}} \frac{\alpha_{C}}{\alpha}\left[1-\left(\frac{\mu}{\Lambda}\right)^{\frac{\alpha}{\alpha_{C}}}\right]<0 .
$$

Thus, we got the condition for chiral symmetry breaking in terms of the dimensionless curvature $\tilde{R} \equiv R / \mu^{2}$. From (33) we see that the critical coupling constant depends on the curvature. For negative curvature the critical coupling is higher and one has a greater chance to find the system in the phase with broken chiral symmetry. As examples of spaces being in correspondence with (34) on can consider the inflationary universe $\mathrm{S}_{4}$ with small curvature or some universe with small constant curvature and a topology of the form $\mathrm{R}_{\mathrm{i}} \times \mathrm{H}_{4-\mathrm{i}}, \mathrm{R}_{\mathrm{i}} \times \mathrm{S}_{4-\mathrm{i}}$, or $\mathrm{S}_{\mathrm{i}} \times \mathrm{H}_{4-\mathrm{i}},(\mathrm{i}=1,2,3)$ (see [27] for explicit examples of quantum field calculations on such backgrounds).

Note that the critical value of the curvature at which symmetry breaking is absent is defined according to (33) by

$$
\frac{R_{C}}{\mu^{2}}=\frac{6 \alpha}{\alpha_{C} g_{4 R}^{*}} .
$$

At all positive curvatures below $R_{c}$ as well as at small negative curvatures the chiral symmetry is broken.

It is quite remarkable that such a simple quantum conditon of chiral symmetry breaking (33), (34) in gauged NJL model in curved spacetime is obtained explicitly. Up to now such a simple symmetry breaking tree-level condition in curved spacetime has been known only for the Higgs sector of (11):

$$
\sigma^{2}=-\left(\xi R+m^{2}\right) / \lambda
$$

where $\xi R+m^{2}<0$. 


\section{Conclusion}

Extending the approach of papers [3], [10] we discussed the gauged NJL model in curved spacetime using quite standard RG language. In this way the RGimproved effective potential for gauged NJL model in curved spacetime is calculated. In the case of fixed gauge coupling $(b \rightarrow+0)$ the condition of chiral symmetry breaking is found explicitly. This condition connects the value of the four-fermion coupling constant with the curvature.

It is interesting to note that one can generalise the results of Section 4 to the case of running gauge coupling too. The only problem to be solved is that even in flat space the effective potential cannot be written explicitly, except in a few simple limiting cases.

To mention also another direction of consideration one can easily apply the above RG approach to study the gauged NJL model in a given specific spacetime like, for example, the DeSitter universe; this may be of importance for the construction of inflationary universes based on composite bound states.

Finally, using the RG approach in the same way one can develop the description of GUT's in curved spacetime via gauged NJL-like models.

SDO would like to thank K. Yamawaki and A.Wipf for useful discussions. This work has been supported by MEC (Spain) and by "Acciones Integradas Hispano-Alemanas".

\section{References}

[1] Y. Nambu and G. Jona-Lasinio, Phys. Rev. 122 (1961) 345; W. A. Bardeen, C. N. Leung and S. T. Love, Phys. Rev. Lett. 56 (1986) 1230

[2] Y. Nambu, in: Proc. Int. Workshop on New Trends in Strong Coupling Gauge Theories, Nagoya, 1988; W. Marciano, Phys. Rev. Lett. 62 (1989) 2723;

[2a] K. Yamawaki, in: Proc. 1990 Int. Workshop on Strong Coupling Gauge Theories, Singapore, 1990; Proc. 1991 Workshop on Effective Field Theories of the Standard Model, Hungary, 1991.

[3] W. Bardeen, C. Hill and M. Lindner, Phys. Rev. D41 (1990) 1647

[4] J. M. Cornwall, R. Jackiw and E. Tomboulis, Phys. Rev. D10 (1974) 2428 
[5] R. W. Haymaker, Riv. Nuovo Cim. 14 (1991) 1

[6] T. Inagaki, T. Muta and S. D. Odintsov, Mod. Phys. Lett. A8 (1993) 2117; E. Elizalde, S. Leseduarte and S. D. Odintsov, Phys. Rev. D49 (1994) 5551; E. Elizalde and S. D. Odintsov, Phys. Rev. D51 (1995) 5990; T. Inagaki, S. Mukaigawa and T. Muta, Phys. Rev. D52 (1995) R4267; E. Elizalde, S. Leseduarte, S. D. Odintsov and Yu. I. Shilnov, Phys. Rev. D 1996; T. Inagaki, preprint HUPD -9522, 1995.

[7] K.-I. Kondo, M. Tanabashi and K. Yamawaki, Progr. Theor. Phys. 89 (1993) 1249; Mod. Phys. Lett. A8 (1993) 2859; K.-I. Kondo, S. Shuto and K. Yamawaki, Mod. Phys. Lett. A6 (1991) 3385.

[8] S. D. Odintsov and Yu. I. Shilnov, Class. Quant. Grav. 8 (1990) 887

[9] C. T. Hill and D. S. Salopek, Ann. Phys. 213 (1992) 21; T. Muta and S. D. Odintsov, Mod. Phys. Lett. A6 (1991) 3641

[10] M. Harada, Y. Kikukawa, T. Kugo and H. Nakano, Progr. Theor. Phys. 92 (1994) 1161

[11] N. P. Chang, Phys. Rev. D10 (1974) 2706; T. P. Cheng, E. Eichten and L.-F. Li, Phys. Rev. D9 (1974) 2259

[12] D. J. Gross and F. Wilczek, Phys. Rev. D8 (1973) 3633; H. D. Politzer, Phys. Rev. Lett. 30 (1973) 1346

[13] N. V. Krasnikov, Mod. Phys. Lett A8 (1993) 797

[14] B. Pendleton and G. Ross, Phys. Lett. B98 (1981) 291

[15] J. Kubo, K. Sibold and W. Zimmermann, Phys. Lett. B220 (1989) 191

[16] B. L. Voronov and I. V. Tyutin, Yad.Fiz. (Sov. J. Nucl. Phys.) 23 (1976) 664

[17] C. T. Hill, C. N. Leung and S. Rao, Nucl. Phys. B262 (1985) 517

[18] I. L. Buchbinder, S. D. Odintsov and I. L. Shapiro, Effective Action in Quantum Gravity, IOP Publishing, Bristol and Philadelphia, 1992

[19] I. L. Buchbinder and S. D. Odintsov, Izv. Vyssh. Uchebn. Zaved. Fiz (Sov.Phys.J.) 12 (1983) 108; Yad. Fiz. 40 (1984) 1338 (Sov. J. Nucl. Phys. 40 (1984) 850); Lett. Nuovo Cim. 42 (1985) 370 
[20] I. L. Buchbinder and S. D. Odintsov, Class. Quant. Grav. 2 (1985) 721; E. Elizalde and S. D. Odintsov, Phys. Lett. B303 (1993) 240; Z.Phys. C64 (1994) 699

[21] A. Hasenfratz, P. Hasenfratz, K. Jansen, J. Kuti and Y. Shen, Nucl. Phys. B365 (1991) 73

[22] F. Cooper, G. Guralnik and N. J. Snyderman, Phys. Rev. Lett. 40 (1978) 1620; G. Guralnik and K. Tamvakis, Phys. Rev. D18 (1978) 4551; J. D. Bjorken, Ann. Phys. (NY) 24 (1963) 174; T. Eguchi, Phys. Rev. D14 (1976) 2755

[23] S. W. Hawking, Talk given on GR-14 Conference, Florence, Italy, 1995

[24] S. Coleman and E. Weinberg, Phys. Rev. D7 (1973) 1888

[25] M. B. Einhorn and D. R. T. Jones, Nucl. Phys. B211 (1983) 29; G. B. West, Phys. Rev. D27 (1983) 1402; K. Yamagishi, Nucl. Phys. B216 (1983) 508; M. Sher, Phys. Repts. 179 (1989) 274; C. Ford, D. R. T.Jones, P.-W. Stevenson and M. B. Einhorn, Nucl. Phys. B395 (1993) 17; M. Bando, T. Kugo, N. Maekawa and H. Nakano, Prog. Theor. Phys. 90 (1993) 405; J. A. Casas, J. R. Espinosa and M. Quiros, Phys. Lett. B342 (1995) 171

[26] W. A. Bardeen and S. T. Love, Phys. Rev. D45 (1992) 4672

[27] E. Elizalde et al, Zeta-Regularization with Applications, World Sci., Singapore, 1994; A. A. Bytsenko, G. Cognola, L. Vanzo and S. Zerbini, Phys.Repts. 1996. 